\title{
Analysis of the transverse effect of Einstein's gravitational waves
}

\section{Christian Corda}

\author{
August 10, 2021 \\ INFN - Sezione di Pisa and Università di Pisa, Via F. Buonarroti 2, I - 56127 \\ PISA, Italy \\ E-mail address: christian.corda@ego-gw.it
}

\begin{abstract}
The investigation of the transverse effect of gravitational waves (GWs) could constitute a further tool to discriminate among several relativistic theories of gravity on the ground. After a review of the TT gauge, the transverse effect of GWs arising by standard general relativity (called Einstein's GWs in this paper) is reanalized with a different choice of coordinates. In the chosen gauge test masses have an apparent motion in the direction of propagation of the wave, while in the transverse direction they appear at rest. Of course, this is only a gauge artefact. In fact, from careful investigation of this particular gauge, it is shown that the tidal forces associated with GWs act along the directions orthogonal to the direction of propagation of waves. In the analysis it is also shown, in a heuristic way, that the transverse effect of Einstein's GWs arises from the propagation of the waves at the speed of the light, thus only massless GWs are purely transverse. But, because the physics of gravitational waves has to be investigated by studing the tidal forces as appearing in the geodesic deviation equation and directly in a laboratory environment on Earth, an analysis of these tidal forces and of the transverse effect in the frame of the local observer is also performed. After this, for a further better understanding of the transverse effect, an example of a wave, which arises from scalar tensor gravity, with both transverse and genuinely longitudinal modes is given and discussed. In the example the connection between the longitudinal component and the velocity of the wave will be mathematical shown.

At the end of this paper the review of the TT gauge is completed, recovering the gauge invariance between the presented gauge and the TT one.
\end{abstract}


PACS numbers: 04.80.Nn, 04.30.Nk, 04.30.-w

\section{Introduction}

The design and construction of a number of sensitive detectors for GWs is underway today. There are some laser interferometers like the VIRGO detector, being built in Cascina, near Pisa by a joint Italian-French collaboration [1, 2], the GEO 600 detector, being built in Hannover, Germany by a joint AngloGermany collaboration [3, 4, the two LIGO detectors, being built in the United States (one in Hanford, Washington and the other in Livingston, Louisiana) by a joint Caltech-Mit collaboration [5, 6], and the TAMA 300 detector, being built near Tokyo, Japan [7, 8]. There are many bar detectors currently in operation too, and several interferometers and bars are in a phase of planning and proposal stages.

The results of these detectors will have a fundamental impact on astrophysics and gravitation physics. There will be lots of experimental data to be analyzed, and theorists will be forced to interact with lots of experiments and data analysts to extract the physics from the data stream.

Detectors for GWs will also be important to confirm or ruling out the physical consistency of general relativity or of any other theory of gravitation [9, 10, 11, 12. This is because, in the context of extended theories of gravity, some differences from general relativity and the others theories can be seen starting by the linearized theory of gravity [9, 10, 12, 13, 14, 15]. In fact, standard Einstein's GWs [18] are in principle different from GWs which arises from others theories of gravity. For example, there are particular extended theories that admit the existence of massive modes of gravitational radiation which can be both scalar and tensorial [9, 12, 13, 14, 15. In this case, the presence of the mass prevents the GW to propagate at the speed of light generating a longitudinal component of the wave (i.e GWs are no more transverse).

The response of interferometers to Einstein's GWs and their transverse effect have been analyzed in lots of works in literature, starting by the work of the Bondi's research group [19] expecially in the TT gauge [20, 21, 22]. With the goal of considering the investigation of the transverse effect of GWs a further tool to discriminate among several relativistic theories of gravity on the ground [12, 13, 14, 15, 16, 17, in this paper, after a review of the TT gauge, the transverse effect of Einstein's GWs is reanalized with a different choice of coordinates. In the chosen gauge test masses have an apparent motion in the direction of propagation of the wave, while in the transverse direction they appear at rest. Of course, this is only a gauge artefact. In fact, from careful investigation of this particular gauge, it is shown that the tidal forces associated with GWs act along the directions orthogonal to the direction of propagation of waves. In the analysis it is also shown, in a heuristic way, that the tranverse effect of Einstein's GWs arises from the propagation of the waves at the speed of light, thus only massless GWs (and this is the case of Einstein's ones) are purely transverse 
(i.e. the presence of the mass precludes GWs to propagate at the speed of the light). But, because the physics of gravitational waves has to be investigated by studing the tidal forces as appearing in the geodesic deviation equation [25] and directly in a laboratory environment on Earth 12, 13, 14, 15, 20, 21, 22, 24, an analysis of these tidal forces and of the transverse effect in the frame of the local observer is also performed, following the lines of [12, 15, 20]. After this, for a further better understanding of the transverse effect, an example of a wave, which arises from scalar tensor gravity, with both transverse and genuinely longitudinal modes is given and discussed [12, 13, 14. In the example the connection between the longitudinal component and the velocity of the wave will be mathematical shown.

At the end of this paper the review of the TT gauge is completed recovering the gauge invariance between the presented gauge and the TT one.

\section{A review of the TT gauge}

Working with $c=1$ and $\hbar=1$, in the context of General Relativity, Einstein field equations can be written like [21, 23]

$$
G_{\mu \nu} \equiv R_{\mu \nu}-\frac{R}{2} \eta_{\mu \nu}=8 \pi \tilde{G} T_{\mu \nu}^{(m)} .
$$

Because we want to study gravitational waves, the linearized theory in vacuum $\left(T_{\mu \nu}^{(m)}=0\right)$ has to be analyzed, with a little perturbation of the background, which is assumed given by the Minkowskian background [21, 23] .

Thus we put

$$
g_{\mu \nu}=\eta_{\mu \nu}+h_{\mu \nu} \text { with }\left|h_{\mu \nu}\right| \ll 1
$$

To first order in $h_{\mu \nu}$, calling $\widetilde{R}_{\mu \nu \rho \sigma}, \widetilde{R}_{\mu \nu}$ and $\widetilde{R}$ the linearized quantity which correspond to $R_{\mu \nu \rho \sigma}, R_{\mu \nu}$ and $R$, the linearized field equations are obtained 12, 15, 21, 23,

$$
\widetilde{R}_{\mu \nu}-\frac{\widetilde{R}}{2} \eta_{\mu \nu}=0
$$

Let us put

$$
\bar{h}_{\mu \nu} \equiv h_{\mu \nu}-\frac{h}{2} \eta_{\mu \nu}
$$

where the inverse transform is the same

$$
h_{\mu \nu}=\bar{h}_{\mu \nu}-\frac{\bar{h}}{2} \eta_{\mu \nu}
$$

By putting eq. (5) in eqs. (3) it is

$$
[] \bar{h}_{\mu \nu}-\partial_{\mu}\left(\partial^{\alpha} \bar{h}_{\alpha \nu}\right)-\partial_{\nu}\left(\partial^{\alpha} \bar{h}_{\alpha \mu}\right)+\eta_{\mu \nu} \partial^{\beta}\left(\partial^{\alpha} \bar{h}_{\alpha \beta}\right),
$$


where [] is the D'Alembertian operator.

Now let us consider the gauge transform (Lorenz condition)

$$
\bar{h}_{\mu \nu} \rightarrow \bar{h}_{\mu \nu}^{\prime}=\bar{h}_{\mu \nu}-\partial_{(\mu} \epsilon_{\nu)}+\eta_{\mu \nu} \partial^{\alpha} \epsilon_{\alpha}
$$

with the condition [] $\epsilon_{\nu}=\partial^{\mu} \bar{h}_{\mu \nu}$ for the parameter $\epsilon^{\mu}$. It is

$$
\partial^{\mu} \bar{h}_{\mu \nu}^{\prime}=0
$$

and, omitting the ', the field equations can be rewritten like

$$
[] \bar{h}_{\mu \nu}=0 .
$$

For a lot of time in literature the condition (7) was been called "Lorentz condition", because physicists have considered its origin due to H. Lorentz (see for example refs. [21, 23]), but its real origin is due to L. Lorenz, see ref. [26]. This particular was explicated in ref. [17, 27] and was communicated to the author of this paper from [28].

Solutions of eqs. (9) are plan waves:

$$
\bar{h}_{\mu \nu}=A_{\mu \nu}(\vec{k}) \exp \left(i k^{\alpha} x_{\alpha}\right)+c . c .
$$

The solutions (10) take the conditions

$$
\begin{aligned}
k^{\alpha} k_{\alpha} & =0 \\
k^{\mu} A_{\mu \nu} & =0,
\end{aligned}
$$
(8).

which arises respectively from the linearized field equations and from eq.

The first of eqs. (11) shows that perturbations have the speed of the light, the second represents the trasverse property of the waves. Here this transverse effect appears from a purely mathematical point of view, but in the next Section we show that this effect is physical.

Fixed the Lorenz gauge, another transformation with []$\epsilon^{\mu}=0$ can be performed; let us take

$$
\begin{gathered}
{[] \epsilon^{\mu}=0} \\
\partial_{\mu} \epsilon^{\mu}=0,
\end{gathered}
$$

which is permissed because [] $\bar{h}=0$. We obtain

$$
\bar{h}=0 \Rightarrow \bar{h}_{\mu \nu}=h_{\mu \nu},
$$

(traceless property) i.e. $h_{\mu \nu}$ is a transverse plane wave too. The gauge transformation (12) also saves the conditions

$$
\begin{gathered}
\partial^{\mu} \bar{h}_{\mu \nu}=0 \\
\bar{h}=0 .
\end{gathered}
$$


Let us consider a wave incoming in the positive $z$ direction; then

$$
k^{\mu}=(k, 0,0 k)
$$

and the second of eqs. (11) implies

$$
\begin{gathered}
A_{0 \nu}=-A_{3 \nu} \\
A_{\nu 0}=-A_{\nu 3} \\
A_{00}=-A_{30}+A_{33} .
\end{gathered}
$$

Now let us compute the freedom degrees of $A_{\mu \nu}$. We was started with 10 components ( $A_{\mu \nu}$ is a symmetric tensor); 3 components have been lost for the trasverse property, more, the condition (13) reduces the components to 6 . One can take $A_{00}, A_{11}, A_{22}, A_{21}, A_{31}, A_{32}$ like independent components; another gauge freedom can be used to put to zero three more components (i.e. one can only chose three of $\epsilon^{\mu}$, the fourth component depends from the others by $\left.\partial_{\mu} \epsilon^{\mu}=0\right)$.

Then, taking

$$
\begin{gathered}
\epsilon_{\mu}=\tilde{\epsilon}_{\mu}(\vec{k}) \exp \left(i k^{\alpha} x_{\alpha}\right)+c . c . \\
k^{\mu} \tilde{\epsilon}_{\mu}=0,
\end{gathered}
$$

the transform law for $A_{\mu \nu}$ is (see eqs. (17) and (10))

$$
A_{\mu \nu} \rightarrow A_{\mu \nu}^{\prime}=A_{\mu \nu}-2 i k\left({ }_{\mu} \tilde{\epsilon}_{\nu}\right) .
$$

Thus, for the six components of interest it is:

$$
\begin{aligned}
& A_{00} \quad \rightarrow \quad A_{00}+2 i k \tilde{\epsilon}_{0} \\
& A_{11} \quad \rightarrow \quad A_{11} \\
& A_{22} \quad \rightarrow \quad A_{22} \\
& A_{21} \quad \rightarrow \quad A_{21} \\
& A_{31} \quad \rightarrow \quad A_{31}-i k \tilde{\epsilon}_{1} \\
& A_{32} \rightarrow A_{32}-i k \tilde{\epsilon}_{2} .
\end{aligned}
$$

The physical components of $A_{\mu \nu}$ are the gauge-invariants $A_{11}, A_{22}$ and $A_{21}$, thus $\tilde{\epsilon}_{\nu}$ can be chosen to put equal to zero the others. From the traceless property it is also $A_{11}=-A_{22}$.

In this way the total perturbation of a gravitational wave propagating in the $z+$ direction in this gauge is

$$
h_{\mu \nu}(t-z)=A^{+}(t-z) e_{\mu \nu}^{(+)}+A^{\times}(t-z) e_{\mu \nu}^{(\times)},
$$

that describes the two polarizations of gravitational waves which arises from General Relativity in the TT gauge (ref. 20, 21, 22]). This gauge is historically called transverse-traceless (TT), because in these particular coordinates the gravitational waves have a transverse effect and are traceless. 


\section{Einstein's GWs in a different gauge}

In the last section we have seen that, in the TT gauge, the perturbation of a plane polarized Einstein's GW propagating in flat spacetime in the $z+$ direction, with a wave front parallel to the $x-y$ plane, is given , considering only the "+" polarization, by

$$
h_{\mu \nu}(t-z)=h(t-z) e_{\mu \nu}^{(+)}
$$

(see also refs. [20, 21, 22]), where $e_{\mu \nu}^{(+)} \equiv \operatorname{diag}(0,1,-1,0)$, the amplitude of the "+" polarization is now labelled $h=h(t-z)$ (i.e. $A^{+}$in previous Section ) and the line element is

$$
d s^{2}=-d t^{2}+d z^{2}+[1+h(t-z)] d x^{2}+[1-h(t-z)] d y^{2} .
$$

A gauge transformation like eq. (17) and the corresponding transformation law (18) can also be used to obtain $A_{00}=A_{22}$. Now the traceless property gives

$$
A_{11}=A_{33}=-A_{00}=-A_{22}
$$

and the line element is

$$
d s^{2}=[1+h(t-z)]\left(-d t^{2}+d x^{2}+d z^{2}\right)+[1-h(t-z)] d y^{2} .
$$

Equation (24) can be also obtained directly from the line element (22) with the substitution

$$
\begin{array}{ccc}
x & \rightarrow & x \\
y & \rightarrow & y \\
z & \rightarrow & z+\frac{1}{2} H(t-z) \\
t & \rightarrow & t-\frac{1}{2} H(t-z),
\end{array}
$$

where

$$
H(t-z) \equiv \int_{-\infty}^{t-z} h(v) d v
$$

In literature it is well known that there exist infinitely many gauge transformations that spoil the TT gauge [21]. The particular interest of the line element (24) arises from the fact that it is a TT gauge of an observer which is moving very slowly in the $z$ direction as it will be shown in the next discussion. In this case it will be also shown that this gauge artefact, that arises in the relativity of motion, will generate a fallacious longitudinal mode of the GW. More, two enlighten relations (eqs. (65) and (66)) will show from an intuitive point of view that the tranverse effect of Einstein's GWs arises from the propagation of the waves at the speed of light. 
Let us see in detail what happens in the gauge (24). The line element can be rewritten as

$$
\left(\frac{d t}{d \tau}\right)^{2}-\left(\frac{d x}{d \tau}\right)^{2}-\left(\frac{d z}{d \tau}\right)^{2}=\frac{1}{1+h}+\frac{1-h}{1+h}\left(\frac{d y}{d \tau}\right)^{2}
$$

where $\tau$ is the proper time of the test masses.

To derive the geodesic equation of motion for test masses (i.e. the beamsplitter and the mirrors of the interferometer) eq. $(87,3 \mathrm{a})$ of [11, which is

$$
\frac{d u_{i}}{d \tau}-\frac{1}{2} \frac{\partial g_{k l}}{\partial x^{i}} u^{k} u^{l}=0,
$$

can be used.

Thus, from the metric (24) it is

$$
\begin{array}{lcc}
\frac{d^{2} x}{d \tau^{2}}= & 0 \\
\frac{d^{2} y}{d \tau^{2}}= & 0 \\
\frac{d^{2} t}{d \tau^{2}}= & \frac{1}{2(1+h)} \partial_{t}(1+h)\left[\left(\frac{d t}{d \tau}\right)^{2}-\left(\frac{d x}{d \tau}\right)^{2}-\left(\frac{d z}{d \tau}\right)^{2}\right]-\frac{1}{2} \partial_{t}(1-h)\left(\frac{d y}{d \tau}\right)^{2} \\
\frac{d^{2} z}{d \tau^{2}}= & -\frac{1}{2(1+h)} \partial_{z}(1+h)\left[\left(\frac{d t}{d \tau}\right)^{2}-\left(\frac{d x}{d \tau}\right)^{2}-\left(\frac{d z}{d \tau}\right)^{2}\right]+\frac{1}{2} \partial_{z}(1-h)\left(\frac{d y}{d \tau}\right)^{2} .
\end{array}
$$

One can immediately integrate the first and the second of eqs. (29) obtaining

$$
\begin{aligned}
& \frac{d x}{d \tau}=C_{1}=\text { const } . \\
& \frac{d y}{d \tau}=C_{2}=\text { const } .
\end{aligned}
$$

Assuming that test masses are at rest initially it is $C_{1}=C_{2}=0$. Thus, even if the GW arrives at test masses, there is not motion of test masses within the $x-y$ plane in this gauge. This can be directly understood from eq. (24) because the absence of the $x$ and of the $y$ dependences in the metric implies that test masses momentum in these directions (i.e. $C_{1}$ and $C_{2}$ respectively) is conserved. This results, for example, from the fact that in this case the $x$ and $y$ coordinates do not explicity enter in the Hamilton-Jacobi equation for a test mass in a gravitational field (see ref. [23]).

Now eq. (27) reads

$$
\left(\frac{d t}{d \tau}\right)^{2}-\left(\frac{d z}{d \tau}\right)^{2}=\frac{1}{1+h} .
$$

In this way, eqs. (29) begins 


$$
\begin{array}{ccc}
\frac{d^{2} x}{d \tau^{2}}= & 0 \\
\frac{d^{2} y}{d \tau^{2}}= & 0 \\
\frac{d^{2} t}{d \tau^{2}}= & \frac{1}{2} \frac{\partial_{t}(1+h)}{(1+h)^{2}} \\
\frac{d^{2} z}{d \tau^{2}}= & -\frac{1}{2} \frac{\partial_{z}(1+h)}{(1+h)^{2}} .
\end{array}
$$

Now it will be shown that, in presence of a GW, a motion of test masses in the $z$ direction, which is the direction of the propagating wave, is present. An analysis of eqs. (33) shows that, to simplify equations, the retarded and advanced time coordinates $(v, w)$ can be introduced:

$$
\begin{gathered}
v=t-z \\
w=t+z .
\end{gathered}
$$

From the third and the fourth of eqs. (33) it is

$$
\frac{d}{d \tau} \frac{d v}{d \tau}=\frac{\partial_{w}[1+h(v)]}{[1+h(v)]^{2}}=0 .
$$

Thus one obtains

$$
\frac{d v}{d \tau}=\alpha
$$

where $\alpha$ is an integration constant. From eqs. (32) and (36), it is

$$
\frac{d w}{d \tau}=\frac{\beta}{1+h}
$$

where $\beta \equiv \frac{1}{\alpha}$, and

$$
\tau=\beta v+\gamma,
$$

where the integration constant $\gamma$ correspondes simply to the retarded time coordinate translation $v=t-z$. Thus, without loss of generality, it can be put equal to zero. Now let us see what is the meaning of the other integration constant $\beta$. The equation for $z$ can be written from eqs. (36) and (37):

$$
\frac{d z}{d \tau}=\frac{1}{2 \beta}\left(\frac{\beta^{2}}{1+h}-1\right) .
$$

When it is $h=0$ (i.e. before the GW arrives at the test masses) eq. (39) becomes

$$
\frac{d z}{d \tau}=\frac{1}{2 \beta}\left(\beta^{2}-1\right)
$$


But this is exactly the initial velocity of the test mass, thus we have to choose $\beta=1$ because we suppose that test masses are at rest initially. This also implies $\alpha=1$.

To find the motion of a test mass in the $z$ direction one can see that from eq. (38) it is $d \tau=d v$, while from eq. (37) it is $d w=\frac{d \tau}{1+h}$. Because it is $z=\frac{w-v}{2}$ we obtain

$$
d z=\frac{1}{2}\left(\frac{d \tau}{1+h}-d v\right)
$$

which can be integrated as

$$
\begin{gathered}
z=z_{0}+\frac{1}{2} \int\left(\frac{d v}{1+h}-d v\right)= \\
=z_{0}-\frac{1}{2} \int_{-\infty}^{t-z} \frac{h(v)}{1+h(v)} d v,
\end{gathered}
$$

where $z_{0}$ is the initial position of the test mass. Now, the displacement of the test mass in the $z$ direction can be written as

$$
\begin{gathered}
\Delta z=z-z_{0}=-\frac{1}{2} \int_{-\infty}^{t-z_{0}-\Delta z} \frac{h(v)}{1+h(v)} d v \\
\simeq-\frac{1}{2} \int_{-\infty}^{t-z_{0}} \frac{h(v)}{1+h(v)} d v .
\end{gathered}
$$

The results can also be rewritten in function of the time coordinate $t$ :

$$
\begin{array}{rcc}
x(t) & = & x_{0} \\
y(t) & = & y_{0} \\
z(t) & = & z_{0}-\frac{1}{2} \int_{-\infty}^{t-z_{0}} \frac{h(v)}{1+h(v)} d(v) \\
\tau(t) & = & t-z(t) .
\end{array}
$$

Then, let us reasume what happens in our gauge: in the $x-y$ plane an inertial test mass initially at rest remains at rest throughout the entire passage of the GW, while in the $z$ direction an inertial test mass initially at rest has a motion during the passage of the GW. This motion has a very weak velocity, in fact from the third of eqs. (44) it is

$$
\frac{d z}{d t} \simeq-\frac{h(t)}{2},
$$

where $\frac{h(t)}{1+h(t)} \simeq h(t)$ has been used .

The following analysis will show that the fallacious effect of this Section is only a gauge artefact. In fact it is well known from the literature that GWs which arises from standard general relativity are longitudinal waves [2, 9, 11, 18, 19, 20, 21, 22, 23, 24, 25. 


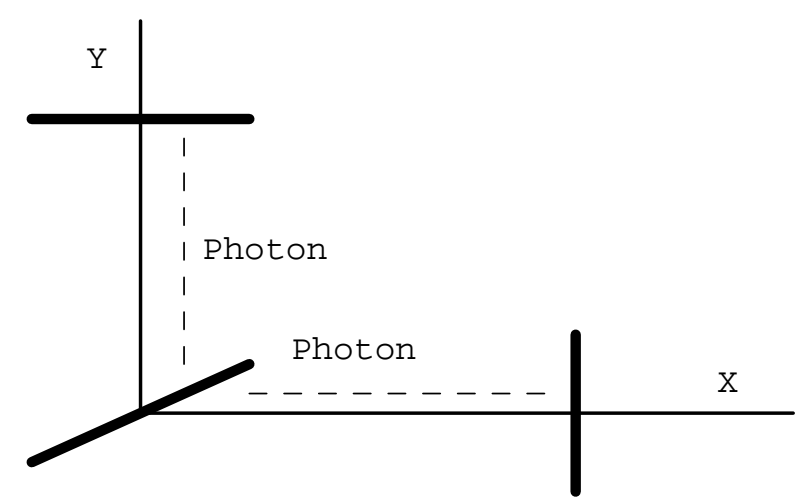

Figure 1: photons can be launched from the beam-splitter to be bounced back by the mirror

\section{Analysis of the transverse effect in the chosen gauge}

The use of words "at rest" has to be clarified: it means that the coordinates of test masses do not change in the presence of the GW in the $x-y$ plane, but we will see that the proper distance between the beam-splitter and the mirror of the interferometer changes even though their coordinates remain the same. On the other hand, it will also be shown that the proper distance between the beam-splitter and the mirror of the interferometer does not change in the $z$ direction even if their coordinates change in the gauge (24).

A good way to analyze variations in the proper distance (time) is by means of "bouncing photons" : a photon can be launched from the beam-splitter to be bounced back by the mirror (see refs. [12, 15, 20] and figure 1).

Let us start by considering the interval for a photon which propagates in the $x$ axis. Assuming that test masses are located along the $x$ axis and the $z$ axis of the coordinate system, the $y$ direction can be neglected because the absence of the $y$ dependence in the metric (24) implies that photon momentum in this direction is conserved 23 and the interval can be rewritten in the form

$$
d s^{2}=[1+h(t-z)]\left(-d t^{2}+d x^{2}+d z^{2}\right) .
$$

Note that photon momentum in the $z$ direction is not conserved, for the $z$ dependence in eq. (24) [20, 23]. Thus photons launched in the $x$ axis will deflect out of this axis. But this effect can be neglected too, because the photon deflection into the $z$ direction will be at most of order $h$ [20]. Then, to first order in $h$, the $d z^{2}$ term can be neglected. Thus, from eq. (46) it is

$$
d s^{2}=(1+h)\left(-d t^{2}\right)+(1+h) d x^{2} .
$$

The condition for null geodesics $\left(d s^{2}=0\right)$ for photons gives 


$$
d t^{2}=d x^{2} .
$$

One recalls that the rate $d \tau$ of the proper time is related to the rate $d t$ of the time coordinate from (ref. [23])

$$
d \tau^{2}=g_{00} d t^{2} .
$$

From eq. (47) it is $g_{00}=(1+h)$. Then, by using eq. (48), one obtains

$$
d \tau^{2}=(1+h) d x^{2},
$$

which gives

$$
d \tau= \pm(1+h)^{\frac{1}{2}} d x .
$$

From eqs. (44) we see that the coordinates of the beam-splitter $x_{b}=l$ and of the mirror $x_{m}=l+L_{0}$ do not change under the influence of the GW in our gauge, thus the proper duration of the forward trip is

$$
\tau_{1}(t)=\int_{l}^{L_{0}+l}[1+h(t)]^{\frac{1}{2}} d x .
$$

To first order in $h$ this integral is approximated by

$$
\tau_{1}(t)=T_{0}+\frac{1}{2} \int_{l}^{L_{0}+l} h\left(t^{\prime}\right) d x
$$

where

$$
t^{\prime}=t-\left(l+L_{0}-x\right) .
$$

In the last equation $t^{\prime}$ is the retardation time (i.e. $t$ is the time at which the photon arrives in the position $l+L_{0}$, so $l+L_{0}-x=t-t^{\prime}$ ) [2, 12, 15, 20].

In the same way, the proper duration of the return trip is

$$
\tau_{2}(t)=T_{0}+\frac{1}{2} \int_{l+L_{0}}^{l} h\left(t^{\prime}\right)(-d x),
$$

where now

$$
t^{\prime}=t-(x-l)
$$

is the retardation time and

$$
T_{0}=L_{0}
$$


is the transit proper time of the photon in absence of the GW, which also corresponds to the transit coordinate time of the photon in presence of the GW (see eq. (48)).

Thus the round-trip proper time will be the sum of $\tau_{2}(t)$ and $\tau_{1}\left(t-T_{0}\right)$. Then, to first order in $h$, the proper duration of the round-trip will be

$$
\tau_{r . t .}(t)=\tau_{1}\left(t-T_{0}\right)+\tau_{2}(t) .
$$

By using eqs. (53) and (54) one sees immediately that deviations of this round-trip proper time (i.e. proper distance) from its imperurbated value are given by

$$
\delta \tau(t)=\frac{1}{2} \int_{l}^{L_{0}+l}\left[h\left(t-2 T_{0}+x-l\right)+h(t-x+l)\right] d x .
$$

The signal seen from the arm in the $x$ axis can be also defined like

$$
\frac{\delta \tau(t)}{T_{0}} \equiv \frac{1}{2 T_{0}} \int_{l}^{L_{0}+l}\left[h\left(t-2 T_{0}+x-l\right)+h(t-x+l)\right] d x .
$$

Now the analysis will be transled in the frequency domain by using the Fourier transform of our field defined by

$$
\tilde{h}(\omega)=\int_{-\infty}^{\infty} d t h(t) \exp (i \omega t) .
$$

By using definition (58), from eq. (57) it is

$$
\frac{\delta \tilde{\tau}(\omega)}{T_{0}}=\Upsilon(\omega) \tilde{h}(\omega),
$$

where $\Upsilon(\omega)$ is the response of the $x$ arm of our interferometer to GWs:

$$
\Upsilon(\omega)=\frac{\exp \left(2 i \omega T_{0}\right)-1}{2 i \omega T_{0}},
$$

which is computed in lots of works in literature, but here the computation has been made in a different gauge.

Now let us see what happens in the $z$ coordinate (see figure 2).

The absences of the $x$ and $y$ dependence in the metric (24) imply that photon momentum in these direction is conserved [23] and the interval can be rewritten in the form

$$
d s^{2}=[1+h(t-z)]\left(-d t^{2}+d z^{2}\right) .
$$

From the condition $d s^{2}=0$ for null geodesics it is

$$
d z= \pm d t
$$

But, from the last of eqs. (44) the proper time is

$$
d \tau(t)=d t-d z,
$$




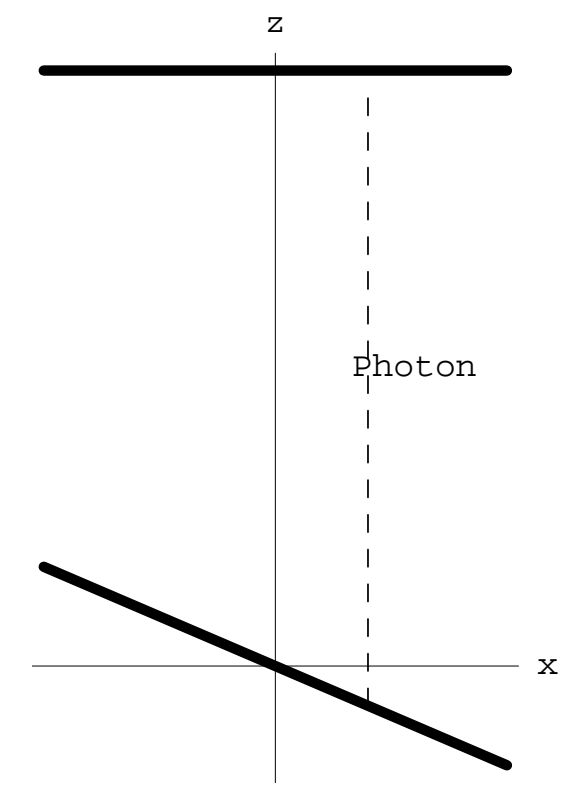

Figure 2: the beam splitter and the mirror are located in the direction of the incoming GW

and, by combining eq. (62) with eq. (63), one gets

$$
d \tau(t)=d t \mp d t .
$$

Thus, it is

$$
\tau_{1}(t)=0
$$

for the forward trip and

$$
\tau_{2}(t)=\int_{0}^{T_{0}} 2 d t=2 T_{0}
$$

for the return trip. Then

$$
\tau(t)=\tau_{1}(t)+\tau_{2}(t)=2 T_{0} .
$$

Thus it is $\delta \tau=\delta L_{0}=0$, i.e. there is not longitudinal effect. This is a consequence of the fact that a GW propagates at the speed of light. Eqs. 650 and (66) show that, in the gauge (24), in the forward trip the photon travels at the same speed of the GW and its proper time is equal to zero ((i.e. the photon and the GW are leaving at the same velocity and in the same direction, giving the result (65)), while in the return trip the photon travels against the GW and 
its proper time redoubles (i.e. the photon and the $\mathrm{GW}$ are leaving at the same velocity, but in opposite direction, giving the result (66) ).

Then it has been shown that the longitudinal effect of eqs. (44) and (45) is fallacious and due to the relativity of motion. The gauge (24) is a TT gauge of an observer which is moving very slowly in the $z$ direction.

\section{Tidal forces and geodesic deviation equations in the gauge of the local observer}

Because the physics of GWs is directly performed in a laboratory environment on Earth [12, 15, 20, 21, 25, the coordinate system in which the space-time is locally flat is typically used and the distance between any two points is given simply by the difference in their coordinates in the sense of Newtonian physics. This gauge is the proper reference gauge of a local observer, located for example in the position of the beam splitter of an interferometer. In this gauge GWs manifest themself by exerting tidal forces on the masses [25] (the mirror and the beam-splitter in the case of an interferometer). A detailed analysis of the frame of the local observer is given in ref. [21], sect. 13.6. Here the more important features of this gauge are recalled:

the time coordinate $x_{0}$ is the proper time of the observer $\mathrm{O}$;

spatial axes are centered in $\mathrm{O}$;

in the special case of zero acceleration and zero rotation the spatial coordinates $x_{j}$ are the proper distances along the axes and the frame of the local observer reduces to a local Lorentz frame: in this case the line element reads [21]

$$
d s^{2}=-\left(d x^{0}\right)^{2}+\delta_{i j} d x^{i} d x^{j}+O\left(\left|x^{j}\right|^{2}\right) d x^{\alpha} d x^{\beta} ;
$$

the effect of the GW on test masses is described by the equation

$$
\ddot{x^{i}}=-\widetilde{R}_{0 k 0}^{i} x^{k},
$$

which is the equation for geodesic deviation in this gauge. The problem is thus

reduced to calculate the linearized Riemann tensor $\widetilde{R}_{0 k 0}^{i}$ in the local gauge of the beam-splitter. But it is well known that $\widetilde{R}_{0 k 0}^{i}$ is gauge invariant [12, 15, 20, 21], thus we can compute it directly from eq. (24). From [21] it is

$$
\widetilde{R}_{\mu \nu \rho \sigma}=\frac{1}{2}\left\{\partial_{\mu} \partial_{\beta} h_{\alpha \nu}+\partial_{\nu} \partial_{\alpha} h_{\mu \beta}-\partial_{\alpha} \partial_{\beta} h_{\mu \nu}-\partial_{\mu} \partial_{\nu} h_{\alpha \beta}\right\}
$$

In this way one obtains,

$$
\begin{gathered}
\widetilde{R}_{010}^{1}=-\frac{1}{2} \ddot{h} \\
\widetilde{R}_{010}^{2}=\frac{1}{2} \ddot{h} \\
\widetilde{R}_{030}^{3}=0 .
\end{gathered}
$$


Using eqs. (69), eqs: (71) give

$$
\begin{aligned}
& \ddot{x}=\frac{1}{2} \ddot{h} x, \\
& \ddot{y}=-\frac{1}{2} \ddot{h} y
\end{aligned}
$$

and

$$
\ddot{z}=0,
$$

which show that the tidal forces act only in a direction perpendicular to the propagating GW, while there is not longitudinal tidal force.

\section{An example of real longitudinal effect in a non - Einsteinian GW}

In this Section it is shown that Scalar-Tensor Gravity, which emerges as "effective theory" from several unification schemes of fundamental interactions (see refs. 10, 12, 13, 14] for a discussion), admits the existence of a scalar massive mode of gravitational waves, where the mass is very small. The mechanism of production is also analyzed, showing that this scalar massive mode has a real (no gauge artefact) longitudinal mode [12, 14]. In this way the correlation between the longitudinal mode and the fact that a GW does not propagate at the speed of light will be made clear also from a mathematical point of view.

Let us ask: what does it mean the term "small"? If one treats scalars like classical waves, that act coherently with the interferometer [12, 13, 14, it has to be $m \ll 1 / L$, where $L=3$ kilometers in the case of Virgo and $L=4$ kilometers in the case of Ligo. Thus it is approximately $m<10^{-9} \mathrm{eV}$. However there is a stronger limitation coming from the fact that the scalar wave should have a frequency which falls in the frequency-range for earth based gravitational antennas that is the interval $10 \mathrm{~Hz} \leq f \leq 10 \mathrm{KHz}$. For a massive $\mathrm{SGW}$ this means:

$$
2 \pi f=\omega=\sqrt{m^{2}+p^{2}},
$$

were $p$ is the momentum. Then it has to be [12, 13, 14]

$$
0 \mathrm{eV} \leq m \leq 10^{-11} \mathrm{eV} .
$$

For these light scalars we can still discuss their effect as a coherent gravitational wave.

In the general context of Scalar-Tensor Gravity [10, 12, 13, 14, Einstein field equations are more general than eq. (11): 


$$
\begin{gathered}
G_{\mu \nu}=-\frac{4 \pi \tilde{G}}{\varphi} T_{\mu \nu}^{(m)}+\frac{\omega(\varphi)}{\varphi^{2}}\left(\varphi_{; \mu} \varphi_{; \nu}-\frac{1}{2} g_{\mu \nu} g^{\alpha \beta} \varphi_{; \alpha} \varphi_{; \beta}\right)+ \\
+\frac{1}{\varphi}\left(\varphi_{; \mu \nu}-g_{\mu \nu}[] \varphi\right)+\frac{1}{2 \varphi} g_{\mu \nu} W(\varphi)
\end{gathered}
$$

with associed a Klein - Gordon equation for the scalar field

$$
[] \varphi=\frac{1}{2 \omega(\varphi)+3}\left(-4 \pi \tilde{G} T^{(m)}+2 W(\varphi)+\varphi W^{\prime}(\varphi)+\frac{d \omega(\varphi)}{d \varphi} g^{\mu \nu} \varphi_{; \mu} \varphi_{; \nu} .\right.
$$

In the above equations $T_{\mu \nu}^{(m)}$ is the ordinary stress-energy tensor of the matter and $\tilde{G}$ is a dimensional, strictly positive, constant [10, 12]. The Newton constant is replaced by the effective coupling

$$
G_{e f f}=-\frac{1}{2 \varphi},
$$

which is, in general, different from $G$. General Relativity is obtained when the scalar field coupling becomes

$$
\varphi=\text { const }=-\frac{1}{2} \text {. }
$$

The case in which it is $\omega=$ const in eqs. (77) and (78) is the stringdilaton gravity [10, 12, 13, 14. Now the linearized theory in vacuum $\left(T_{\mu \nu}^{(m)}=\right.$ 0 ) has to be studied in a little different perturbation of the background with respect standard Einstein's GW. The scalar field $\varphi=\varphi_{0}$ has to be added to the Minkowskian background [10, 12]. We also assume $\varphi_{0}$ to be a minimum for $W$ :

$$
W \simeq \frac{1}{2} \alpha \delta \varphi^{2} \Rightarrow W^{\prime} \simeq \alpha \delta \varphi
$$

The linearization of equations is parallel to the canonical one of thesecond Section of this paper, thus one can put

$$
\begin{gathered}
g_{\mu \nu}=\eta_{\mu \nu}+h_{\mu \nu} \\
\varphi=\varphi_{0}+\delta \varphi .
\end{gathered}
$$

To first order in $h_{\mu \nu}$ and $\delta \varphi$, the linearized field equations now are [9, 12]

$$
\begin{aligned}
\widetilde{R}_{\mu \nu}-\frac{\widetilde{R}}{2} \eta_{\mu \nu} & =\partial_{\mu} \partial_{\nu} \Phi+\eta_{\mu \nu}[] \Phi \\
{[] \Phi } & =m^{2} \Phi,
\end{aligned}
$$

where we have defined

$$
\begin{aligned}
\Phi & \equiv-\frac{\delta \varphi}{\varphi_{0}} \\
m^{2} & \equiv \frac{\alpha \varphi_{0}}{2 \omega+3} .
\end{aligned}
$$


In analogy to the purely General Relativity case of Section 2, the linearized Riemann tensor and the linearized eqs. (83) are invariant for gauge transformations

$$
\begin{gathered}
h_{\mu \nu} \rightarrow h_{\mu \nu}^{\prime}=h_{\mu \nu}-\partial_{(\mu} \epsilon_{\nu)} \\
\delta \varphi \rightarrow \delta \varphi^{\prime}=\delta \varphi ;
\end{gathered}
$$

then one can define

$$
\bar{h}_{\mu \nu} \equiv h_{\mu \nu}-\frac{h}{2} \eta_{\mu \nu}+\eta_{\mu \nu} \Phi,
$$

and, considering the gauge transform (Lorenz condition) with the condition

$$
[] \epsilon_{\nu}=\partial^{\mu} \bar{h}_{\mu \nu}
$$

for the parameter $\epsilon^{\mu}$, it is

$$
\partial^{\mu} \bar{h}_{\mu \nu}=0 .
$$

Thus, the field equations like can be rewritten

$$
\begin{gathered}
{[] \bar{h}_{\mu \nu}=0} \\
{[] \Phi=m^{2} \Phi .}
\end{gathered}
$$

Solutions of eqs. (89) and (90) are plan waves:

$$
\begin{aligned}
\bar{h}_{\mu \nu} & =A_{\mu \nu}(\vec{p}) \exp \left(i p^{\alpha} x_{\alpha}\right)+c . c . \\
\Phi & =a(\vec{p}) \exp \left(i q^{\alpha} x_{\alpha}\right)+c . c .
\end{aligned}
$$

where

$$
\begin{aligned}
k^{\alpha} & \equiv(\omega, \vec{p}) & \omega & =p \equiv|\vec{p}| \\
q^{\alpha} & \equiv\left(\omega_{m}, \vec{p}\right) & \omega_{m} & =\sqrt{m^{2}+p^{2}} .
\end{aligned}
$$

In eqs. (89) and (91) the equation and the solution for the tensorial waves exactly like in General Relativity have been obtained (Section 2), while eqs. (90) and (92) are respectively the equation and the solution for the massive scalar mode.

Note: now the dispersion law for the modes of the massive scalar field $\Phi$ is not linear [12, 14, 15. The velocity of every tensorial mode $\bar{h}_{\mu \nu}$ is the light speed $c$, but the dispersion law (the second of eq. (93) for the modes of $\Phi$ is that of a massive field which can be discussed like a wave-packet [12, 14, 15]. Also, the group-velocity of a wave-packet of $\Phi$ centered in $\vec{p}$ is 


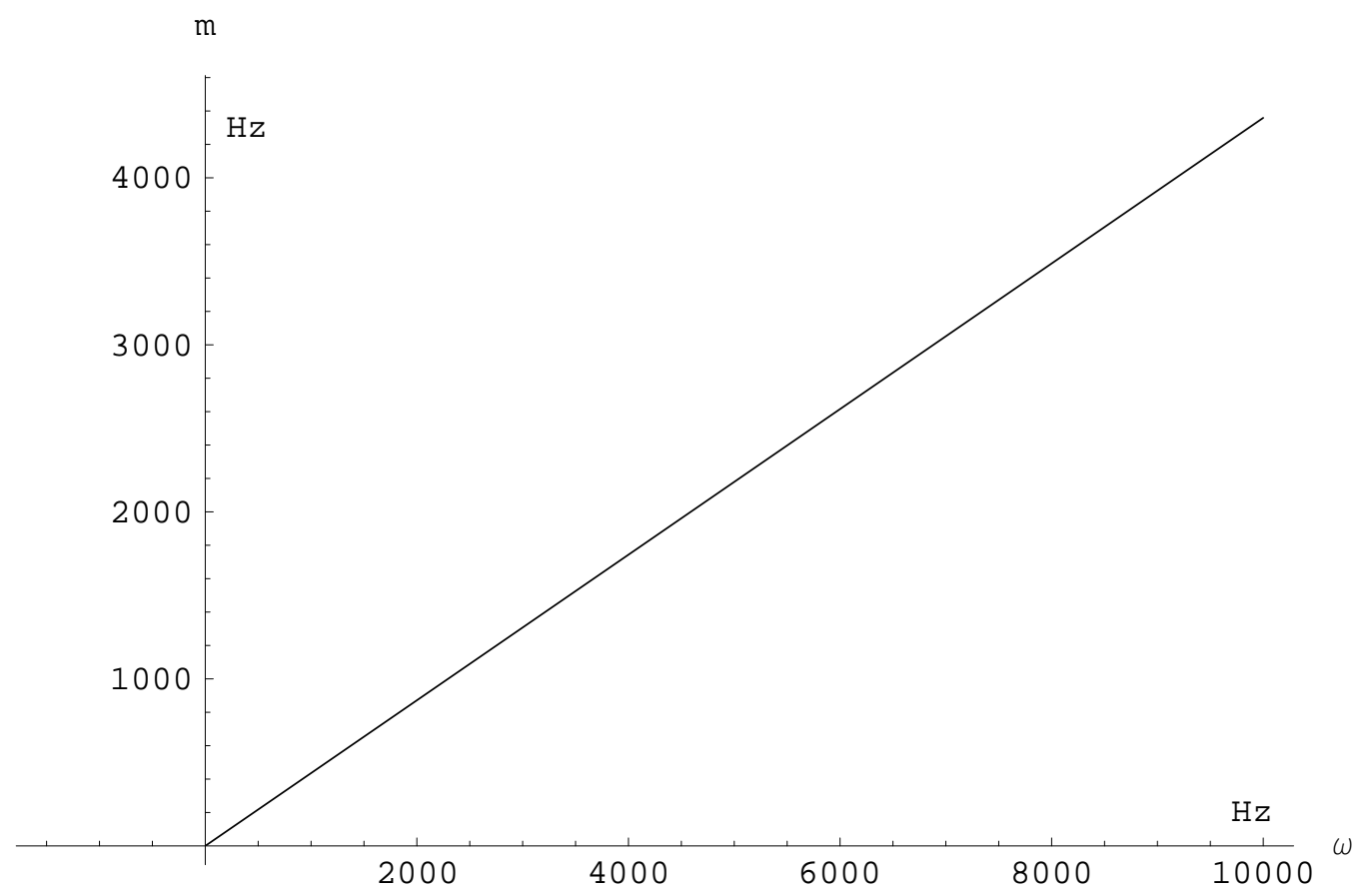

Figure 3: from Capozziello S and Corda C - Int. J. Mod. Phys. D 151119 -1150 (2006), the mass-frequency relation for a massive SGW incoming with a speed of $0.9 c$ : for the mass it is $1 \mathrm{~Hz}=10^{-15} \mathrm{eV}$

$$
\overrightarrow{v_{G}}=\frac{\vec{p}}{\omega}
$$

which is exactly the velocity of a massive particle with mass $m$ and momentum $\vec{p}$.

From the second of eqs. (93) and eq. (94) it is simple to obtain [12, 14]:

$$
v_{G}=\frac{\sqrt{\omega^{2}-m^{2}}}{\omega} .
$$

Then, wanting a constant speed of our wave-packet, one needs

$$
m=\sqrt{\left(1-v_{G}^{2}\right)} \omega .
$$

The relation (96) is shown in fig. 3 for a value $v_{G}=0.9$.

Now let us remain in the Lorenz gauge with trasformations of the type []$\epsilon_{\nu}=0$; this gauge gives a transverse condition for the tensorial part of the field: $k^{\mu} A_{\mu \nu}=0$, but we do not know if the total field $h_{\mu \nu}$ is transverse [15]. From eq. (86) it is: 


$$
h_{\mu \nu}=\bar{h}_{\mu \nu}-\frac{\bar{h}}{2} \eta_{\mu \nu}+\eta_{\mu \nu} \Phi .
$$

At this point, being in the massless case one could put

$$
\begin{gathered}
{[] \epsilon^{\mu}=0} \\
\partial_{\mu} \epsilon^{\mu}=-\frac{\bar{h}}{2}+\Phi,
\end{gathered}
$$

which gives the transverse condition of the total field. But in the massive case this is impossible. In fact, applying the D'Alembertian operator to the second of eqs. (98) and using the field equations (89) and (90) it is

$$
[] \epsilon^{\mu}=m^{2} \Phi,
$$

which is in contrast with the first of eqs. (98). In the same way it is possible to show that it does not exist any linear relation between the tensorial field $\bar{h}_{\mu \nu}$ and the scalar field $\Phi$. Thus a gauge in wich $h_{\mu \nu}$ is purely spatial cannot be chosen (i.e. we cannot put $h_{\mu 0}=0$, see eq. (97)). But one can put the traceless condition to the field $\bar{h}_{\mu \nu}$ :

$$
\begin{gathered}
{[] \epsilon^{\mu}=0} \\
\partial_{\mu} \epsilon^{\mu}=-\frac{\bar{h}}{2},
\end{gathered}
$$

which imply

$$
\partial^{\mu} \bar{h}_{\mu \nu}=0 .
$$

Wanting to save the conditions $\partial_{\mu} \bar{h}^{\mu \nu}$ and $\bar{h}=0$, one can use transformations like

$$
\begin{gathered}
{[] \epsilon^{\mu}=0} \\
\partial_{\mu} \epsilon^{\mu}=0,
\end{gathered}
$$

and, taking $\vec{p}$ in the $z$ direction, it is possible to choose a gauge in which only $A_{11}, A_{22}$, and $A_{12}=A_{21}$ are different to zero. The condition $\bar{h}=0$ gives $A_{11}=-A_{22}$. Now, putting these equations in eq. (97) it is

$$
h_{\mu \nu}(t, z)=A^{+}(t-z) e_{\mu \nu}^{(+)}+A^{\times}(t-z) e_{\mu \nu}^{(\times)}+\Phi\left(t-v_{G} z\right) \eta_{\mu \nu} .
$$

The term $A^{+}(t-z) e_{\mu \nu}^{(+)}+A^{\times}(t-z) e_{\mu \nu}^{(\times)}$describes the two standard (i.e. tensorial) polarizations of gravitational waves which arises from General Relativity, exactly like in Section 2, while the term $\Phi\left(t-v_{G} z\right) \eta_{\mu \nu}$ is the massive scalar field.

Now it will be shown that the discussion of the physical effect of the wave is different from the massless case [12, 13, 14. 
For a pure scalar gravitational wave equation (103) becomes

$$
h_{\mu \nu}(t, z)=\Phi\left(t-v_{G} z\right) \eta_{\mu \nu},
$$

and, with an analysis parallel to the one of previous Section, using equation (103) directly in equations (70), for the gauge invariance of the linearized Riemann tensor, one gets in the frame of the local observer

$$
\begin{aligned}
& \widetilde{R}_{010}^{1}=-\frac{1}{2} \ddot{\Phi} \\
& \widetilde{R}_{010}^{2}=-\frac{1}{2} \ddot{\Phi} \\
& \widetilde{R}_{030}^{3}=\frac{1}{2}[] \Phi .
\end{aligned}
$$

But, putting the field equation (90) in the third of eqs. (105) it is

$$
\widetilde{R}_{030}^{3}=\frac{1}{2} m^{2} \Phi,
$$

which shows that the field is not transversal.

Infact, using eq. (69) it results

$$
\begin{aligned}
\ddot{x} & =\frac{1}{2} \ddot{\Phi} x, \\
\ddot{y} & =\frac{1}{2} \ddot{\Phi} y
\end{aligned}
$$

and

$$
\ddot{z}=-\frac{1}{2} m^{2} \Phi(t, z) z .
$$

Then the effect of the mass is the generation of a longitudinal force (in addition to the transverse one).

For a better understanding of this longitudinal force, let us analyse the effect on test masses in the context of the geodesic deviation.

Following [14] one puts

$$
\widetilde{R}_{0 j 0}^{i}=\frac{1}{2}\left(\begin{array}{ccc}
-\partial_{t}^{2} & 0 & 0 \\
0 & -\partial_{t}^{2} & 0 \\
0 & 0 & m^{2}
\end{array}\right) \Phi(t, z)=-\frac{1}{2} T_{i j} \partial_{t}^{2} \Phi+\frac{1}{2} L_{i j} m^{2} \Phi .
$$

Here we have used the transverse projector with respect to the direction of propagation of the GW $\widehat{n}$, defined by

$$
T_{i j}=\delta_{i j}-\widehat{n}_{i} \widehat{n}_{j},
$$

and the longitudinal projector defined by 


$$
L_{i j}=\widehat{n}_{i} \widehat{n}_{j} .
$$

In this way the geodesic deviation equation (69) can be rewritten like

$$
\frac{d^{2}}{d t^{2}} x_{i}=\frac{1}{2} \partial_{t}^{2} \Phi T_{i j} x_{j}-\frac{1}{2} m^{2} \Phi L_{i j} x_{j} .
$$

Thus one sees immediately that the effect of the mass present in the GW generates a longitudinal force proportional to $\mathrm{m}^{2}$ which is in addition to the transverse one. But if $v_{g} \rightarrow 1$ in eq. (96) it is $m \rightarrow 0$, and the longitudinal force vanishes. Thus it is clear that the longitudinal mode arises from the fact that the scalar part of the GW does no propagate at the speed of light.

In ref. [12 the analysis has been generalized to all the frequencies (eqs. (110) and (113) are correct only in the low frequencies approximation) with the computation of the longitudinal response function of interferometers for massive scalar GW, which is (eq. (136) of [12])

$$
\begin{gathered}
\Upsilon_{l}(\omega)=\left(1-\frac{1}{v_{P}^{2}}\right) \exp \left[i \omega\left(1+\frac{1}{v_{P}}\right) L\right]+\frac{v_{P}\left(1-\frac{1}{v_{P}^{2}}\right)}{4 L \omega}\left(\frac{\exp [2 i \omega L]\left(i v_{P}^{2}-\left(v_{P}-1\right) v_{P} \omega+i L\left(v_{P}-1\right)^{2} \omega^{2}\right.}{\left(v_{P}-1\right)^{3}}+\right. \\
\frac{2 \exp \left[i \omega\left(1+\frac{1}{v_{P}}\right) L\right]\left(-2 i v_{P}^{2}\left(3 v_{P}^{2}+1\right)+2(1+L) v_{P}\left(v_{P}^{4}-1\right) \omega+i L^{2}\left(v_{P}+1\right)^{2} \omega^{2}\right)}{\left(v_{P}^{2}-1\right)^{3}}+ \\
\left.-\frac{2 i v_{P}^{2}+2 v_{P}\left(v_{P}+1\right) \omega+2 i L\left(v_{P}+1\right)^{2} \omega^{2}}{\left(v_{P}+1\right)^{3}}\right),
\end{gathered}
$$

and for $m \rightarrow 0$ (i.e. $v_{P} \rightarrow 1$ ) one gets $\Upsilon_{l}(\omega) \rightarrow 0$.

In figure 4 the longitudinal response function of the Virgo interferometer to two massive scalar GWs with speeds of $0.1 c$ (non relativistic case) and $0.9999 c$ (ultra relativistic case, thick line) is shown. In the non-relativistic case the longitudinal signal is strong while in the ultra relativistic case (i.e. $v_{P} \rightarrow 1$ ) is very weak.

\section{A control of gauge invariance: computation in the TT gauge for Einstein's GWs}

In this Section, returning in the context of standard Einstein's GWs, a brief review of the portion of ref. 20], which analyzes the TT gauge, is given for a control of gauge invariance that shows the correctness of the results.

Let us start by considering the interval for a photon which propagates in the $x$ axis. With the same considerations about the conservation of the photon momentum, one can write eq. (22) as

$$
d s^{2}=-d t^{2}+[1+h(t-z)] d x^{2} .
$$

Putting the condition $d s^{2}=0$ for null geodesics in the line element (115) the coordinate velocity of the photon is obtained 


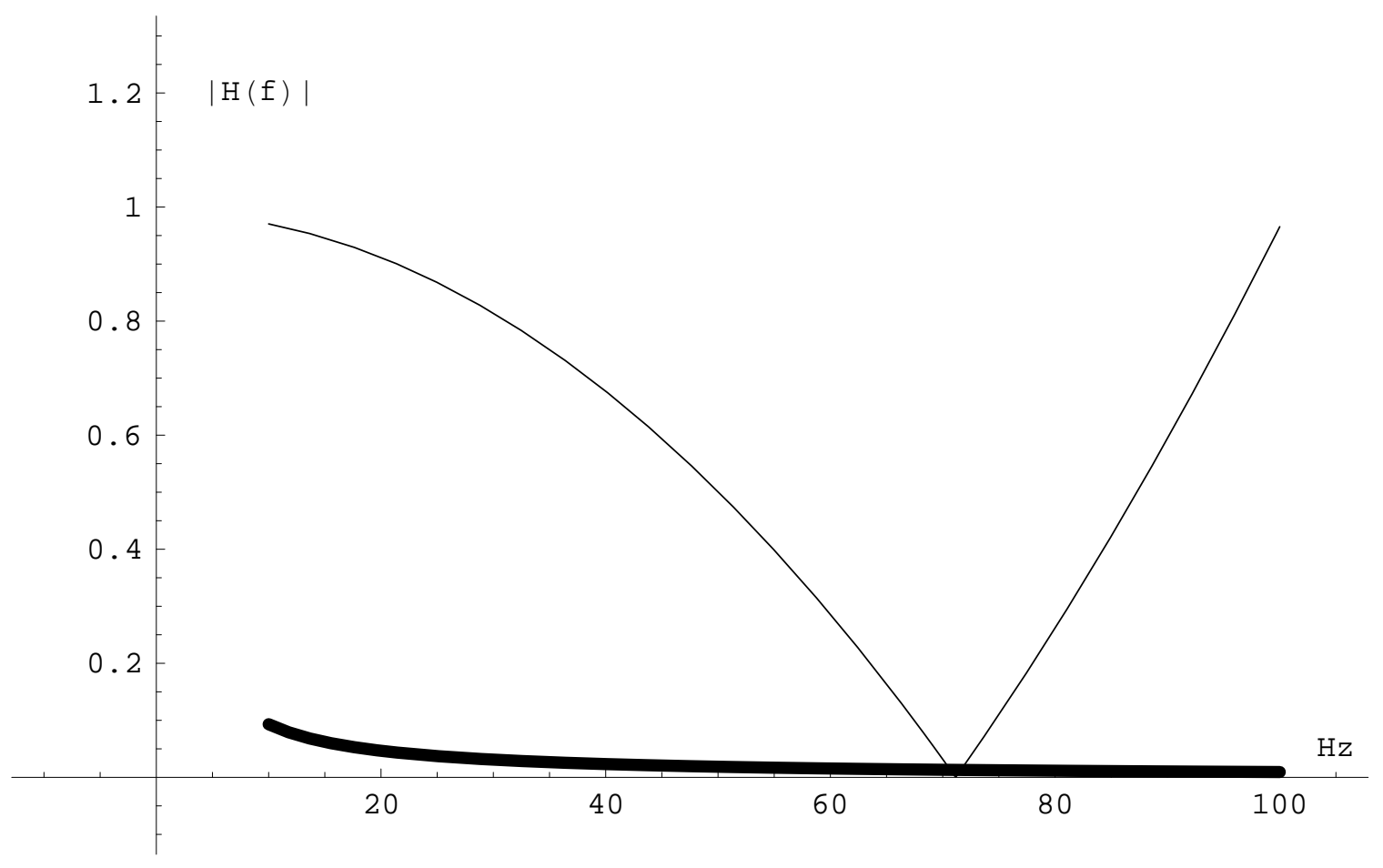

Figure 4: from Capozziello S and Corda C - Int. J. Mod. Phys. D 151119 1150 (2006), the absolute value, at low frequencies, of the longitudinal response function of the Virgo interferometer to two SGWs with speeds of $0.1 c$ (non relativistic case) and $0.9999 c$ (ultra relativistic case, thick line). 


$$
v^{2} \equiv\left(\frac{d x}{d t}\right)^{2}=\frac{1}{1+h(t)},
$$

which is a convenient quantity for calculations of the photon propagation time between the beam-splitter and the mirror [2, 12, 15, 20]. In the TT gauge, the time coordinate $t$ is also the proper time at every point of the space, because $g_{00}=1$ [21, 23].

In this gauge the coordinates of the beam-splitter $x_{b}=l$ and of the mirror $x_{m}=l+L_{0}$ do not changes under the influence of the GW [2, 20, thus one can find the duration of the forward trip as

$$
T_{1}(t)=\int_{l}^{L_{0}+l} \frac{d x}{v\left(t^{\prime}\right)},
$$

with

$$
t^{\prime}=t-\left(l+L_{0}-x\right)
$$

where in the last equation $t^{\prime}$ is the retardation time.

To first order in $h$ this integral can be approximated with

$$
T_{1}(t)=T_{0}+\frac{1}{2} \int_{l}^{L_{0}+l} h\left(t^{\prime}\right) d x,
$$

where

$$
T_{0}=L_{0}
$$

is the transit time of the photon in absence of the GW. Similiary, the duration of the return trip will be

$$
T_{2}(t)=T_{0}+\frac{1}{2} \int_{l+L_{0}}^{l} h\left(t^{\prime}\right)(-d x),
$$

though now the retardation time is

$$
t^{\prime}=t-(x-l) .
$$

Equations (118) and (119) are exactly equal to equations (53) and (54). Then, the same computation that has been made in Section 4 can be performed in this case too, obtaining

$$
\delta T(t)=\frac{1}{2} \int_{l}^{L_{0}+l}\left[h\left(t-2 L_{0}+x-l\right)+h(t-x+l)\right] d x .
$$

Thus, in the $x$ direction of the TT gauge, there is the same variation of proper time (distance) of eq. (56), which was obtained in the $x$ direction of the different gauge analyzed in Section 4.

For a photon which propagates in the $z$ axis the analysis is trivial. The condition $d s^{2}=0$ for null geodesic gives now 


$$
d t= \pm d z .
$$

Then, by recalling that $t$ is the proper time in this gauge, it is:

$$
\tau(t)=T_{0}+T_{0}=2 T_{0} .
$$

Thus it is $\delta \tau=\delta L_{0}=0$, i.e. a longitudinal effect is not present.

By confronting eq. (120) with eq. (56) and eq. (122) with eq. (67) the gauge invariance between our gauge and the TT one is recovered.

\section{Conclusions}

Because the investigation of the transverse effect of gravitational waves (GWs) could constitute a further tool to discriminate among several relativistic theories of gravity on the ground, after a review of the TT gauge, the transverse effect of Einstein's GWs has been reanalized with a different choice of coordinates. In the chosen gauge test masses have an apparent motion in the direction of propagation of the wave, while in the transverse direction they appear at rest. Of course, this is only a gauge artefact. In fact, from careful investigation of this particular gauge, it has been shown that the tidal forces associated with GWs

act along the directions orthogonal to the direction of propagation of waves. In the analysis it has also been shown, in a heuristic way, that the tranverse effect of Einstein's GWs arises from the propagation of the waves at the speed of light, thus only massless GWs (and this is the case of Einstein's ones) are purely transverse (i.e. the presence of the mass precludes GWs to propagate at the speed of the light). But, because the physics of gravitational waves has to be investigated by studing the tidal forces as appearing in the geodesic deviation equation and directly in a laboratory enviroment on Earth, an analysis of these tidal forces and of the transverse effect in the frame of the local observer has also been performed. After this, for a further better understanding of the transverse effect, an example of a wave, which arises from scalar tensor gravity, with both transverse and genuinely longitudinal modes has been given and discussed. In the example the connection between the longitudinal component and the velocity of the wave has been mathematical shown.

At the end of this paper the review of the TT gauge has been completed, recovering the gauge invariance between the presented gauge and the TT one.

\section{Acknowledgements}

I would like to thank Salvatore Capozziello, Mauro Francaviglia, Maria Felicia De Laurentis and Giancarlo Cella for helpful advices during my work. It is a pleasure to thank Giampiero Esposito for clarifications about the Lorenz gauge. I thank the referee for precious advices and comments that allowed to improve this paper. The European Gravitational Observatory (EGO) consortium has also to be thanked for the using of computing facilities. 


\section{References}

[1] Acernese F et al. (the Virgo Collaboration) Class. Quantum Grav. 23, No 19, S635-S642 (2006)

[2] Corda C - Astropart. Phys. 27, No 6, 539-549 (2007)

[3] Hild S (for the LIGO Scientific Collaboration ) Class. Quantum Grav. 23, No 19 S643-S651 (2006)

[4] Willke B et al. Class. Quantum Grav. 23, No 8, S207-S214 (2006)

[5] Sigg D (for the LIGO Scientific Collaboration ) www.ligo.org/pdf_public/P050036.pdf

[6] Abbott B et al. ( the LIGO Scientific Collaboration ) - Phys. Rev. D 72, $042002(2005)$

[7] Ando M and the TAMA Collaboration Class. Quantum Grav. 19, No 7, $1615-1621$ (2002)

[8] Tatsumi D, Tsunesada Y and the TAMA Collaboration Class. Quantum Grav. 21, No 5, S451-S456 (2004)

[9] Capozziello S - Newtonian Limit of Extended Theories of Gravity in Quantum Gravity Research Trends Ed. A. Reimer, pp. 227-276 Nova Science Publishers Inc., NY (2005) - also in arXiv:gr-qc/0412088 (2004)

[10] Capozziello S and Troisi A - Phys. Rev. D 72044022 (2005)

[11] Will C M Theory and Experiments in Gravitational Physics, Cambridge Univ. Press Cambridge (1993)

[12] Capozziello S and Corda C - Int. J. Mod. Phys. D 151119 1150 (2006); Corda C - Response of laser interferometers to scalar gravitational waves- talk in the Gravitational Waves Data Analysis Workshop in the General Relativity Trimester of the Institut Henri Poincare - Paris 13-17 November 2006, on the web in www.luth2.obspm.fr/IHP06/workshops/gwdata/corda.pdf

[13] Tobar ME, Suzuki T and Kuroda K Phys. Rev. D 59102002 (1999)

[14] Maggiore M and Nicolis A - Phys. Rev. D 62024004 (2000)

[15] Corda C - J. Cosmol. Astropart. Phys. JCAP04009 (2007)

[16] Corda C - Astropart. Phys. doi:10.1016/j.astropartphys.2007.05.009

[17] Alemanni G, Francaviglia M, Ruggiero ML and Tartaglia A - Gen. Rev. Grav. 3711 (2005); Alemanni G, Capone M, Capozziello S and Francaviglia M - Gen. Rev. Grav. 381 (2006) 
[18] Einstein A - Uber Gravitationswellen, Sitz Preuss. Akad. Wiss. 8 154-167 (1918)

[19] Bondi H, Pirani F A E and Robinson I - Proc. Roy. Soc. Lond. A251 519-533 (1959)

[20] Rakhmanov M - Phys. Rev. D 71084003 (2005)

[21] Misner CW, Thorne KS and Wheeler JA - "Gravitation" - W.H.Feeman and Company - 1973

[22] Maggiore M- Physics Reports 331 283-367 (2000)

[23] Landau L and Lifsits E - "Teoria dei campi" - Editori riuniti edition III (1999)

[24] Saulson PR - " Fundamentals of interferometric gravitational wave detectors" - World Scientific, Singapore - New Jersey - London - Hong Kong (1994)

[25] Private Communication with the referee

[26] Lorenz L Phil. Mag. 34, 287 (1867)

[27] Penrose R and Rindler W - "Spinors and spacetime" - vol. 1 Cambridge University Press (1984)

[28] Private Communication with Prof. Giampiero Esposito, of Naples University 